\title{
CÓMO SURGE Y SE VINCULA EL CONOCIMIENTO RELACIONADO CON EL DESEMPEÑO EN LAS PYMES: UN ANÁLISIS CUALITATIVO
}

\author{
How knowledge relatedness arises and is linked to performance in SMEs: a qualitative study
}

Rafael Ángel Villafuerte Godínez

Administrador de Empresas. Instituto Tecnológico de Costa Rica, Cartago - Costa Rica, rafavilla91@gmail.com

\section{Juan Carlos Leiva}

Doctor en Dirección de Empresas. Instituto Tecnológico de Costa Rica, Cartago - Costa Rica, jleiva@itcr.ac.cr

\section{Cómo citar/ How to cite}

Villafuerte, R. y Leiva, J. (2015). Cómo surge y se vincula el conocimiento relacionado con el desempeño en las Pymes: un análisis cualitativo. Revista CEA, 1(2), 37-48.

Recibido: 15 de mayo de 2015

Aceptado: 25 de junio de 2015

\section{Resumen}

El presente artículo busca describir cómo se relacionan el conocimiento y el desempeño en las pequeñas y medianas empresas, bajo el enfoque teórico de conocimiento relacionado y en un contexto de un país en vías de desarrollo, como Costa Rica. El conocimiento relacionado (knowledge relatedness) se entiende como el grado de similitud que existe entre el conocimiento que utiliza un emprendedor en su propia empresa con respecto al utilizado en su trabajo en una empresa progenitora, es decir la empresa de la cual salió de trabajar el emprendedor al fundar la propia. El estudio se realizó mediante una investigación cualitativa, en la cual se entrevistaron ocho emprendedores provenientes de firmas multinacionales los cuales actualmente cuentan con su propio emprendimiento. Se halló que las variables influyentes en el surgimiento del conocimiento relacionado son tres (puesto que desempeñó en la multinacional, tipo de emprendimiento formado y momento de incorporación al emprendimiento). Además en las tres posturas planteadas para desempeño se halló una relación positiva con respecto a la utilización del conocimiento relacionado.

Palabras clave: conocimiento relacionado, Pyme, desempeño empresarial, habilidades.

\begin{abstract}
This report seeks to describe how knowledge and performance can be related on small and medium size enterprises, using the approach of knowledge relatedness and in the context of a developing country like Costa Rica. Knowledge relatedness is defined as the degree of similarity between the knowledge of a company related to an original company, in other words, the company which the entrepreneur left in order to establish their own company. The study was conducted using qualitative research, in which eight entrepreneurs, from multinational companies, were interviewed, which now have their own businesses. It was found that the influential variables in the emergence of relatedness knowledge are three (position in the multinational, SME type and time of incorporation). Also in the three positions referred to performance, a positive relationship was found regarding the use of related knowledge.
\end{abstract}

Keywords: knowledge relatedness, SMEs, business performance, skills. 


\section{INTRODUCCIÓN}

En la literatura científica (Davidsson y Honig, 2003; Dencker, et al., 2009, Noel y West, 2009) se puede apreciar una discrepancia interesante sobre la relación entre conocimiento relacionado y desempeño de los nuevos emprendimientos, entendiendo el conocimiento relacionado como el grado de similitud que existe entre el conocimiento que utiliza un emprendedor en su propia organización con respecto al utilizado en su trabajo en una empresa progenitora, es decir, la empresa de la cual salió de trabajar el emprendedor al fundar la propia. Unas posturas señalan que esa relación es positiva, a mayor conocimiento relacionado mejor desempeño de los emprendimientos (Davidsson y Honig, 2003; Dencker, et al., 2009) mientras que la otra señala que no hay relación. (Noel y West, 2009).

En muchos países en vías de desarrollo, la atracción de inversión extranjera directa de empresas multinacionales (EMN) es un pilar de sus estrategias de desarrollo. Con la llegada de estas EMN se busca incrementar la competitividad, generar empleo, producir encadenamientos y obtener efectos de derrame (externalidades positivas) sobre el resto de la economía (Spencer, 2008).

Ligando lo anterior con el conocimiento relacionado, recientemente Leiva et al., (2014) no encontraron diferencias significativas entre el desempeño de empresas creadas por emprendedores con experiencia previa como empleados en empresas multinacionales versus otros emprendedores sin ese tipo de experiencia laboral previa.

Es a partir de esos antecedentes donde la presente investigación pretende aportar. El objetivo es indagar, con una perspectiva cualitativa, qué tipo de conocimientos adquieren los emprendedores en su paso previo como empleados de las EMN y su utilidad para el éxito de su propio emprendimiento. Esto por cuanto una de las principales necesidades en los países en vías de desarrollo hoy día es generar conocimiento que coadyuve en la creación y la sostenibilidad de nuevas empresas.

Es así como el artículo presenta una intersección temática poco tratada en la literatura: conocimiento relacionado, efectos derrame de la atracción de EMN y desempeño de los nuevos emprendimientos.

\section{MARCO TEÓRICO}

Existen tres enfoques teóricos principales sobre lo que es conocimiento relacionado. El primero lo conceptualiza como la influencia del conocimiento aplicado en la empresa derivada proveniente de su empresa progenitora, siendo la empresa progenitora la empresa en la cual solía trabajar el emprendedor (Sapienza, et al., 2009; Noel y West, 2009; Leiva, et al., 2014). El segundo lo define como la transferencia de conocimientos provenientes de organizaciones externas y su utilización para crear una ventaja competitiva (Weber y Weber, 2010; Brush y Vanderwerf, 1992). A la vez que el tercero lo define como el intercambio de conocimientos entre negocios y compañías multinacionales (Hansen, 2002, Tariverd y Venkatraman, 2005). En este artículo se asume el primer enfoque.

Otra de las variables desarrolladas en el estudio es la que corresponde al desempeño, de igual manera hay diversidad de enfoques teóricos. Un primer enfoque lo define como la probabilidad que tienen las organizaciones de operar con eficiencia en el mercado (Zahra, et al., 2002). Una segunda perspectiva lo define como la construcción que pueda realizar una organización de sus ventajas competitivas sostenibles en el tiempo (Leiva, et al., 2014; Gruber, 2007; Noel y West, 2009). Un tercer concepto lo ve como el desempeño empresarial, la rentabilidad económica de la organización y el aumento en ventas (Lopéz e Iglesias, 2006; Brush y Vanderwerf, 1992; Sapienza, et al., 2009). 
Por otra parte, en cuanto a los efectos derrame de la atracción de EMN sobre las pymes locales existen diferentes criterios. Uno de estos indica que las relaciones con las EMN son positivas para las pymes, esto debido a que sostener dichas relaciones va ligado con un aumento de sus ventas, así como en la creación de productos de mayor calidad, la implementación de nuevas tecnologías, nuevos enfoques de mercado, entre otros (Muñoz, et al., 2012; Spencer, 2008).

En el sentido inverso, el ingreso de las EMN en el mercado no siempre tiene un efecto positivo. Al ser estas organizaciones más desarrolladas y posicionadas tienden a llevarse el mejor capital humano, dejando a las pymes sin personal capacitado, el cual es esencial para su desarrollo. A la vez que muchas veces llegan a sectores donde las pymes no están suficientemente desarrolladas, imposibilitando la creación de alianzas entre organizaciones y con mucha más razón una sana competencia (Spencer, 2008; Castellani y Zanfei, 1998).

Enfocado en el ámbito costarricense, donde se efectuó el estudio de campo, se dice que emprendedores provenientes de EMN, suelen en su mayoría adquirir habilidades blandas como negociación, comunicación, trabajo duro, motivación entre otras. Sin embargo, en lo atinente a las habilidades duras o estructurales y de manejo de la organización (como lo son mercadotecnia, estrategia de negocios generales, y logística de operaciones) los emprendedores confesaron que las estrategias en sus emprendimientos eran muy diferentes en comparación a las que habían visualizado en las multinacionales, por lo cual no les es fácil aplicar ese conocimiento adquirido (Monge, et al., 2011; Monge, et al., 2012).

Finalmente, en cuanto a la relación entre el conocimiento relacionado y el desempeño empresarial, investigaciones previas muestran diferentes posturas.

Una de las posturas sostiene que ese conocimiento previo aumenta la probabilidad de supervivencia de la organización, incluso afirma que las empresas con bajos niveles de conocimiento previo tienen el doble de probabilidades de fracasar, en comparación a la organización que sí lo posee (Dencker, et al., 2009; Clercq y Arenius, 2006). Viendo el desempeño como aumento de ventas e ingresos, se afirma que el conocimiento previo adquirido por el emprendedor tiene relación con el rendimiento que pueda obtener su nueva empresa (Weber y Weber, 2010; Tariverd y Venkatraman, 2005).

En contraposición, la otra postura afirma que el conocimiento previo adquirido no tiene impacto con el desempeño de la organización (Leiva, et al., 2014; Noel y West, 2009). En este caso, percibiendo el desempeño, como la construcción que pueda realizar una organización de sus ventajas competitivas sostenibles en el tiempo.

En esta línea, concretamente en un análisis más ligado al que presenta nuestro artículo, Leiva et al., (2014) concluyen que si bien la experiencia en una EMN puede ser de utilidad en un emprendimiento, no existe ninguna diferencia superior en el desempeño empresarial, medida en términos, tanto de crecimiento en las ventas como en el rendimiento financiero de las firmas creadas por exempleados de EMN respecto de las creadas por emprendedores no exempleados de EMN.

\section{RESULTADOS Y DISCUSIÓN}

El estudio se efectuó en Costa Rica, país reconocido por su exitosa estrategia de atracción de inversión extranjera directa (World Economic Forum, 2013). Es de tipo cualitativo ya que se enfoca en comprender y profundizar los fenómenos, explorándolos desde la perspectiva de los participantes en un ambiente natural y en la relación con el contexto, a la vez que se utilizó el diseño de la teoría fundamentada, esto debido a que el propósito de esta, es desarrollar teoría basada en datos y se aplica a áreas específicas. (Hernández, et al., 2010). 
Al ser un estudio cualitativo, las muestras están representadas por grupos de personas, eventos, sucesos, comunidades, con el fin de recolectar los datos necesarios, y que estos sean representativos de la población que se vaya a estudiar. No se utilizó ningún método estadístico ya que la muestra no es probabilística.

En cuanto a la técnica de investigación se utilizó la entrevista, debido a que es más íntima, flexible y abierta (Hernández, et al., 2010). En concreto se utilizó como instrumento de investigación una entrevista semiestructurada con guía de preguntas o asuntos, a la vez que se le permitió al entrevistador introducir preguntas adicionales para precisar conceptos $u$ obtener mayor información sobre el tema. En lo correspondiente al análisis de datos cabe destacar lo siguiente: en primer lugar, se le dio estructura a los datos, se describieron y analizaron las experiencias de las personas estudiadas, se comprendieron contextos; seguidamente se procedió a explicar fenómenos y por último se relacionaron los resultados con la teoría estudiada previa al estudio.

Las entrevistas fueron realizadas a emprendedores que laboraron o laboran en una empresa multinacional, y actualmente tienen su propio emprendimiento. Por ello se decidió utilizar la muestra caso tipo, la cual es muy utilizada, donde el objetivo es la riqueza, profundidad y calidad de la información (Hernández, et al., 2010). Para la selección de dichos emprendedores se utilizó el criterio de expertos ligados al fomento del emprendimiento y la incubación de empresas en Costa Rica. Entre los entrevistados se encuentran personas que ocuparon puestos gerenciales, así como puestos operativos. Hay quienes pasaron todo el proceso de ascenso desde un puesto operativo a una gerencia, así como quienes a su llegada a la multinacional se incorporaron directamente a un puesto gerencial. También el grupo de entrevistados presenta diversidad de profesiones, entre las cuales se hallan: administradores, ingenieros y una farmacéutica. Es importante recalcar la existencia de variedad de género, específicamente siete hombres y una mujer. A la vez que se incluyen personas cuyo tipo de emprendimiento varía, entre los cuales se tiene de productos, servicios y comercio.

A continuación se presenta la Tabla 1, la cual muestra de una forma más concisa lo mencionado anteriormente.

Tabla 1. Perfil de los emprendedores

Table 1. Entrepreneurs Profile

\begin{tabular}{|c|c|c|c|c|}
\hline Entrevistado & Profesión & Nombre de la empresa & $\begin{array}{c}\text { Tipo de } \\
\text { emprendimiento }\end{array}$ & $\begin{array}{c}\text { Multinacional en la } \\
\text { que laboró }\end{array}$ \\
\hline Ricardo Coto Kith & Administrador & Intercasa & Productos & Walmart \\
\hline Carlos Sirias Quesada & Ingeniero & Pernix & Servicios & Intel \\
\hline Pablo Zeledón Hidalgo & Administrador & $\begin{array}{l}\text { Negocios varios de } \\
\text { entrenamiento }\end{array}$ & Comercio & $P \& G$ \\
\hline Juliana Redondo Zúñiga & Farmacéutica & Cetratec- $\alpha$ & Servicios & Calox \\
\hline William Jaubert Solano & Administrador & LT Trades & Servicios & $\begin{array}{l}\text { British American } \\
\text { Tobacco }\end{array}$ \\
\hline Julio Cortés Mixter & Ingeniero & Golden Supply Chain Co. & Servicios & Walmart \\
\hline $\begin{array}{c}\text { Pedro Cascante } \\
\text { Mondragón \& Maicol } \\
\text { Calderón Mondragón }\end{array}$ & $\begin{array}{l}\text { Publicista \& } \\
\text { Financista }\end{array}$ & Positive Crossfit & Servicios & Intel \& Amazon \\
\hline
\end{tabular}

Fuente: Elaboración propia 
En seguida se mostrarán los resultados por medio de secciones. Primero las variables que influyen la presencia del conocimiento relacionado, seguido de la influencia del conocimiento en las posturas de rendimiento, y finaliza con la influencia de la multinacional en la creación del emprendimiento.

\section{Variables que influyen la presencia del conocimiento relacionado}

Esta sección muestra los principales resultados producto del análisis de las entrevistas. Dicha información mostró que en general, la presencia del conocimiento relacionado en un emprendimiento va a depender de las siguientes variables.

La primer variable es el puesto en el cual se desempeñó el emprendedor en la multinacional, ya que según comentaban los entrevistados, a mayor sea el grado del puesto que desempeñó el emprendedor en la multinacional, así va a ser el conocimiento o el tipo de habilidades que se le desarrollan al emprendedor en esta entidad. Los entrevistados comentaban que mientras mayor fuese el grado o el puesto que desempeñaban, las habilidades blandas eran las que las multinacionales buscaban desarrollar en ellos, estos productos se vuelven más estratégicos, y no tanto de operación, como sucedía cuando trabajaban en puestos de menor rango $u$ operativos, donde la multinacional buscaba desarrollar principalmente sus habilidades duras.

En una de las entrevistas se comentó:

Tuve la oportunidad de ocupar varios cargos e ir creciendo conforme me fui desarrollando en la empresa, y en los puestos más bajos u operativos me desarrollaban más las habilidades duras en comparación de las blandas y conforme fui creciendo y empecé a ocupar puestos más altos y gerenciales, la organización me desarrollaba más las blandas (Redondo, 2015).

La segunda variable es el tipo de emprendimiento que realice el emprendedor versus el fin o las actividades que desarrolla la multinacional en la cual trabajaba. Esto porque el tipo conocimiento que se aplica en una empresa de comercio, de servicios o de productos suele variar, al igual que las habilidades que se desarrollan o se adquieren en las multinacionales van a variar según el fin o la misión que esta tenga. Es importante también considerar el lugar de proveniencia de esta, ya que dependiendo de dónde provenga la multinacional su cultura organizacional varía, así como el conocimiento y el tipo de habilidades que busca y desarrolla en sus colaboradores.

En una de las entrevistas, Zeledón (2015) comenta que la multinacional en la cual trabajó le desarrolló ciertas habilidades, las cuales iban en función al puesto que desempeñaba, sin embargo cuando realizó su emprendimiento, no consideró que el conocimiento adquirido en dicha organización fuera de utilidad para el desarrollo de esto, debido a que las funciones que realizaba en la multinacional no tenían ninguna relación con su emprendimiento, por lo cual no utilizó ese conocimiento adquirido en la empresa progenitora.

Por otra parte, Jaubert (2015) considera que su paso por la multinacional fue de gran utilidad pues le permitió desarrollar habilidades, las cuales son vitales para su emprendimiento y el desarrollo de este, ya que las funciones que realizaba en la empresa que laboraba están muy ligadas con una de las principales funciones que realiza en su emprendimiento, e incluso le llegó a permitir, conocer y saber cómo manejar a quienes serían sus clientes.

La tercera variable a considerar es sí el emprendedor empieza su organización desde cero, o si bien se incorpora a un emprendimiento ya establecido, debido a que el tiempo de incorporación a la Mipyme puede variar por el tipo y el grado de aparición del conocimiento relacionado en la organización a la cual se incorpora.

En otra de las entrevistas, uno de los consultados que se incorporó al emprendimiento, ya 
establecido, señaló que la utilización del conocimiento adquirido es de uso diario, sin embargo al principio fue algo más de enfoque administrativo, control contable, liderar el negocio y, conforme iba pasando el tiempo, se va enfocando más en la estrategia y en la consecución del negocio: buscar negocio, crear negocio (Coto, 2015). Caso contrario al de los otros entrevistados, los cuales empezaron su emprendimiento desde cero y mencionaban que la utilización de las habilidades blandas, al inicio de su emprendimiento, fue esencial en aspectos como la conquista de clientes y proveedores, y que incluso llegaron a utilizar más estas, en comparación de las habilidades determinadas duras (Cortés; Jaubert; Redondo Zeledón, 2015).
En cuanto a las habilidades adquiridas, varían dependiendo de la multinacional y el puesto que desempeñó el emprendedor. Entre las principales desarrolladas se encuentran: comunicación, orden, enfoque en objetivos y resultados, dinamismo, manejo de tecnologías y cumplimiento de objetivos. (Cortés; Coto; Jaubert; Redondo; Zeledón; 2015).

El tipo de variables halladas están en línea con hallazgos similares de otros trabajos en ámbitos similares (Leiva et al., 2014; Monge et al., 2011).

A continuación se presenta la Tabla 2, la cual muestra de una forma más concisa lo mencionado anteriormente.

Tabla 2. Determinación y aplicación de habilidades

Table 2. Determination and application of skills

\begin{tabular}{|c|c|c|c|c|}
\hline Entrevistado & $\begin{array}{l}\text { Tipo de } \\
\text { empresa }\end{array}$ & $\begin{array}{l}\text { Habilidades que aprendió } \\
\text { en la multinacional }\end{array}$ & $\begin{array}{l}\text { Habilidades que } \\
\text { utilizó al inicio del } \\
\text { emprendimiento }\end{array}$ & $\begin{array}{c}\text { Habilidades que } \\
\text { utiliza actualmente } \\
\text { en su } \\
\text { emprendimiento }\end{array}$ \\
\hline Ricardo Coto Kith & Productos & $\begin{array}{l}\text {-Enfocarse en los } \\
\text { resultados } \\
\text {-No atacar personas y a } \\
\text { atacar problemas } \\
\text {-Análisis financiero } \\
\text {-Formas y técnicas de } \\
\text { análisis } \\
\text {-Definir rumbo } \\
\text { organizacional } \\
\text {-Consistencia con sus } \\
\text { objetivos } \\
\text {-Cómo hacer } \\
\text { presentaciones efectivas } \\
\text {-Percepción de las } \\
\text { habilidades adquiridas } \\
80 \% \text { Habilidades duras } \\
20 \% \text { habilidades blandas }\end{array}$ & $\begin{array}{l}\text { Duras más que } \\
\text { blandas. }\end{array}$ & $\begin{array}{c}\text { Blandas más que } \\
\text { duras. }\end{array}$ \\
\hline $\begin{array}{l}\text { Carlos Sirias } \\
\text { Quesada }\end{array}$ & Servicios & No puede determinar & No puede determinar & $\begin{array}{l}\text { No puede } \\
\text { determinar }\end{array}$ \\
\hline $\begin{array}{l}\text { Pablo Zeledón } \\
\text { Hidalgo }\end{array}$ & Comercio & $\begin{array}{l}\text {-Habilidades de } \\
\text { comunicación } \\
\text {-Desarrollo del inglés } \\
\text {-Percepción de las } \\
\text { habilidades adquiridas } \\
\text { 80\% habilidades blandas } \\
20 \% \text { habilidades duras }\end{array}$ & Blandas & Blandas \\
\hline
\end{tabular}




\begin{tabular}{|c|c|c|c|c|}
\hline $\begin{array}{c}\text { Juliana Redondo } \\
\text { Zúñiga }\end{array}$ & Servicios & $\begin{array}{l}\text {-Habilidades Técnicas } \\
\text {-Comunicación } \\
\text {-Manejo TIC } \\
\text {-Liderazgo }\end{array}$ & Blandas y Duras & $\begin{array}{c}\text { Blandas más que } \\
\text { duras }\end{array}$ \\
\hline $\begin{array}{l}\text { William Jaubert } \\
\text { Solano }\end{array}$ & Servicios & $\begin{array}{l}\text {-Técnicas } \\
\text {-Herramientas } \\
\text {-Manejo de tecnologías } \\
\text {-Conocer estructuras de } \\
\text { las transnacionales } \\
\text {-Comunicación } \\
\text {-Liderazgo } \\
60 \% \text { Duras } \\
\text { 40\% Blandas }\end{array}$ & Blandas & Blandas y Duras \\
\hline Julio Cortés Mixter & Servicios & $\begin{array}{l}\text {-Orden } \\
\text {-Visión } \\
\text {-Enfocarse en los } \\
\text { resultados } \\
\text {-Manejo organizacional } \\
\text {-Mantener la estrategia } \\
\text {-Seguir objetivos } \\
\text {-Ansiedad de venta } \\
\text {-Ansiedad de crecimiento } \\
\text {-Saber segmentar } \\
\text {-Entender a sus clientes } \\
\text {-Administrar el talento } \\
\text {-BenchMarking } \\
\text {-Dinamismo } \\
\text { 30\% Duras } \\
\text { 70\% Blandas }\end{array}$ & $\begin{array}{c}\text { Blandas más que } \\
\text { duras }\end{array}$ & Blandas y duras \\
\hline $\begin{array}{l}\text { Pedro Cascante } \\
\text { Mondragón \& } \\
\text { Maicol Calderón } \\
\text { Mondragón }\end{array}$ & Servicios & $\begin{array}{l}\text {-Planificar } \\
\text {-Promoción } \\
\text {-Control } \\
\text {-Re planeación } \\
\text {-Ejecución } \\
\text {-Atención al cliente } \\
\text {-Buscar mercados } \\
\text {-Disciplina } \\
\text {-Responsabilidad } \\
\text {-Ambición } \\
\text {-Paciencia } \\
\text {-Dinamismo } \\
\text {-Percepción de habilidades } \\
\text { adquiridas } \\
\text { 50\% Duras } \\
\text { 50\% Blandas }\end{array}$ & Blandas y duras & Blandas y duras \\
\hline
\end{tabular}

Fuente: Elaboración propia 


\section{Influencia del conocimiento en las posturas de desempeño}

En esta sección se muestra la influencia del conocimiento relacionado en el desempeño de los emprendimientos. Es importante señalar que para la variable desempeño, se plantearon las tres posturas establecidas en el marco teórico.

Para la primera postura, los entrevistados, en su mayoría, opinaron que dicho conocimiento influyó en la probabilidad de operar con eficiencia en el mercado de sus organizaciones, tal y como menciona uno de los entrevistados, «la multinacional, tiene varias características, de planeación estratégica de monitoreo y de cronograma de trabajo. Características que si aplica en su empresa aumenta la probabilidad de ser eficiente» (Jaubert, 2015).

Así las cosas, la influencia del conocimiento relacionado en esta perspectiva de desempeño organizacional concuerda con lo que menciona Dencker,et al., (2009, p. 525) y Clercq y Arenius (2006, p. 350), en sus investigaciones: el conocimiento previo adquirido tiene un impacto directo en el nivel de supervivencia de las organizaciones.

En la segunda postura, algunos de los entrevistados determinaron que dicho conocimiento sí influyó en la creación de ventajas competitivas sostenibles en el tiempo. Sin embargo, aquellos que consideran que no hubo una influencia en la creación de ventajas competitivas sostenibles en el tiempo es por casos como que su emprendimiento no está relacionado con el conocimiento adquirido en la multinacional, y las funciones que realizaba en dicha entidad son completamente divergentes a las que realiza actualmente en su organización, o porque considera que al tener la multinacional un enfoque de mercado muy diferente al cual se enfoca en su emprendimiento, no le permitió adquirir conocimiento para la creación de dichas ventajas competitivas.
Sin embargo, la mayoría afirma que dicho conocimiento fue de gran influencia a la hora de la creación de sus ventajas competitivas, caso contrario a las posturas que sostienen Leiva, et al., (2014, p.130 ), así como Noel y West (2009, p.15), quienes en sus investigaciones sostienen que dicho conocimiento previo no tiene impacto positivo sobre esta postura de desempeño planteada.

En la tercera postura, la mayoría de los entrevistados concordaron en que dicho conocimiento sí influyó en la rentabilidad económica de la organización y aumentó las ventas. Sin embargo, aquellos que consideran que no hubo una influencia en dicha postura, es producto de que su emprendimiento no está relacionado con el conocimiento adquirido en la multinacional, y las funciones que realizaba en dicha entidad son completamente opuestas a las que realiza actualmente en su organización.

Por lo que se podría considerar que sí hay cierta influencia del conocimiento relacionado con el desempeño desde el punto de vista de la tercera postura planteada. Al igual que lo establecen Weber y Weber (2010, p. 29); Tariverd y Venkatraman, (2005, p.112 ) en sus investigaciones, donde afirman que el conocimiento previo adquirido por el emprendedor tiene relación con el rendimiento que pueda obtener este en su organización.

Es importante recalcar que en comparación de los diferentes autores citados, estos en su mayoría evaluaron el conocimiento previo como un todo, caso diferente al de esta investigación, en la cual se evaluó el conocimiento exclusivamente adquirido en el paso de los emprendedores en una multinacional, lo cual podría variar positiva o negativamente la pecepción de la influencia del conocimiento en el desempeño.

A continuación se presenta la Tabla 3, la cual muestra de una forma más concisa lo mencionado anteriormente. 
Tabla 3. Relación del conocimiento en el desempeño

Table 3. Relationship between knowledge and performance

\begin{tabular}{llcccc|}
\hline & \multicolumn{4}{c}{$\begin{array}{c}\text { Influencia del conocimiento en las } \\
\text { postura de desempeño }\end{array}$} \\
\hline \multicolumn{1}{|c}{ Entrevistado } & $\begin{array}{c}\text { Tipo de } \\
\text { empresa }\end{array}$ & I Postura & II Postura & II Postura \\
\hline Ricardo Coto Kith & Productos & Sí & Sí & Sí \\
\hline Carlos Sirias Quesada & Servicios & $\begin{array}{c}\text { No puede } \\
\text { determinar }\end{array}$ & $\begin{array}{c}\text { No puede } \\
\text { determinar }\end{array}$ & $\begin{array}{c}\text { No puede } \\
\text { determinar }\end{array}$ \\
\hline Pablo Zeledón Hidalgo & Comercio & No & No & No \\
\hline $\begin{array}{l}\text { Juliana Redondo } \\
\text { Zúniga }\end{array}$ & Servicios & Sí & No & Sí \\
\hline $\begin{array}{l}\text { William Jaubert } \\
\text { Solano }\end{array}$ & Servicios & Sí & Sí & Sí \\
\hline $\begin{array}{l}\text { Julio Cortés Mixter } \\
\text { Pedro Cascante }\end{array}$ & Servicios & Sí & Sí & Sí \\
\hline $\begin{array}{l}\text { Mondragón \& Maicol } \\
\text { Calderón Mondragón }\end{array}$ & Servicios & Sí & Sí & Sí \\
\hline
\end{tabular}

Fuente: Elaboración propia

\section{Influencia de la multinacional en la creación de los emprendimientos}

En este tópico, los emprendedores coinciden en que su paso por la multinacional fue un gran apoyo y, a la vez, un puente en la elaboración de su emprendimiento. Manifiestan que su paso por ésta fue esencial en aspectos como: creación de capital de trabajo, experiencia y creación de redes de contacto.

Por ejemplo, Cascante y Calderón (2015) comentan que a pesar de que ellos tienen su propio emprendimiento, aún no está lo suficientemente desarrollado, de tal manera que les permita poder vivir exclusivamente de la rentabilidad de este, por esa razón continúan ligados a la empresa multinacional. Sin duda alguna, la mayoría del capital creado para la inversión en su organización es proveniente de las remuneraciones brindadas por la multinacional e incluso sirve de gran apoyo en los momentos que el emprendimento ha necesitado inyección de capital.

Caso similar al de Sirias (2015), quien indica, que si bien no puede determinar la aparición del conocimento relacionado en la creación en su emprendimiento, así como la influencia que este pudo tener en el desempeño de su organización, al igual que Cascante y Calderón (2015) coincide que el capital adquirido en la multinacional fue de gran apoyo para la creación de su emprendimiento.

Para enfocar los principales resultados obtenidos se presenta el siguiente mapa conceptual. 


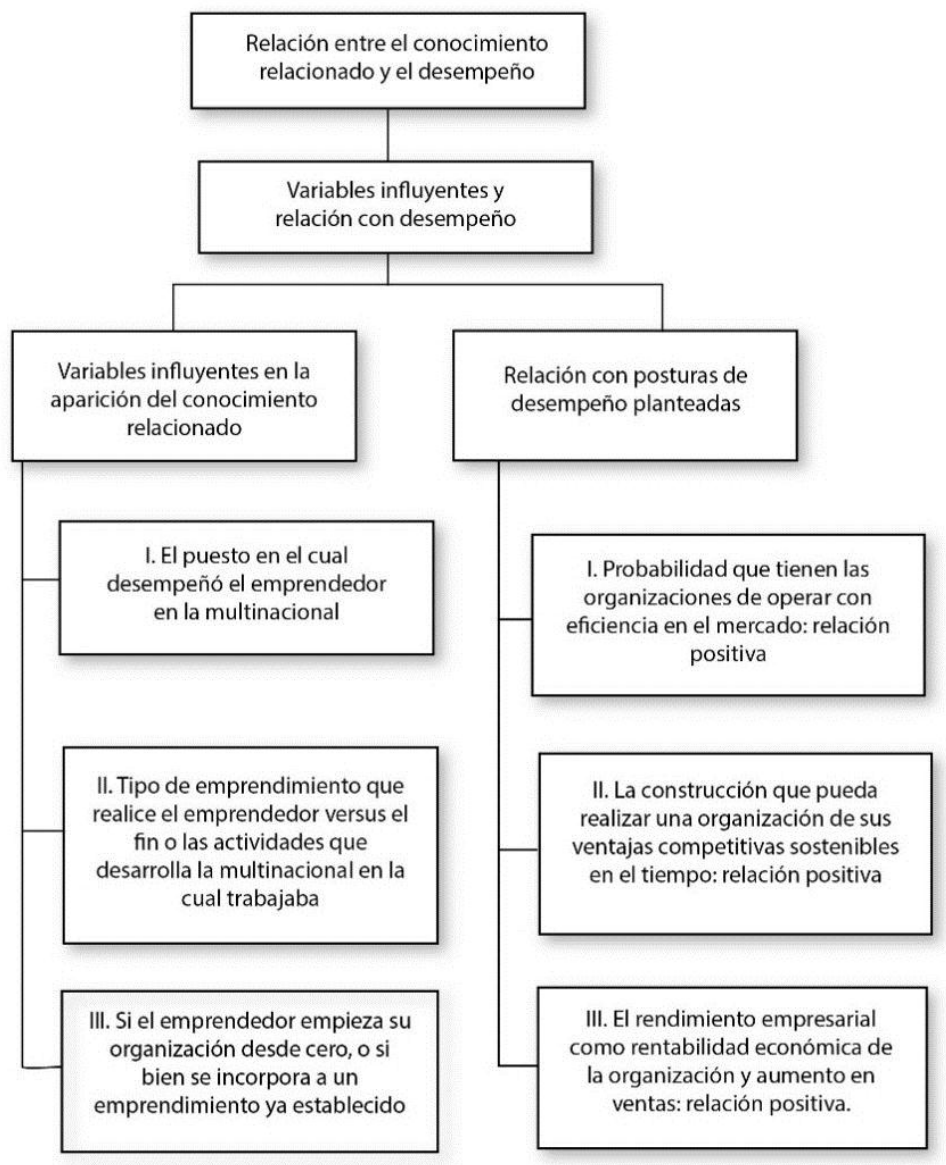

Figura 1. Mapa conceptual de principales hallazgos del estudio

Figure 1. Conceptual map of main findings

Fuente: Elaboración propia

\section{CONCLUSIONES}

Esta investigación tuvo como objetivo describir cómo se relacionan concretamente el conocimiento y el desempeño en las Pymes, bajo el enfoque de conocimiento relacionado y en un contexto como el costarricense, que es un país latinoamericano en vía de desarrollo.

En su mayoría, los emprendedores manifestaron que su paso por las empresas multinacionales fue de gran ayuda para su desarrollo, tanto profesional como personal. Además, el conocimiento previo adquirido se convirtió en un eslabón esencial en la construcción de su emprendimiento, a tal punto que la aparición de dicho conocimiento fue vital para el crecimiento de sus organizaciones, así como en el desempeño de estas.

Se determinó que la aparición del conocimiento relacionado va a depender de tres variables principales: el puesto en el cual se desempeñó el emprendedor en la multinacional, el tipo de emprendimiento que realice versus el fin o las actividades que desarrolla la multinacional en la cual trabajaba y si empieza su organización desde cero, o si bien se incorpora a un emprendimiento ya establecido.

Esto debido a que según el comportamiento del emprendedor en cada una de las variables, así a va ser la aparición del conocimiento relacionado en sus organizaciones, y por ende, la influencia 
que este pueda tener en el desempeño de los emprendimientos.

Es importante mencionar que en el desempeño de los emprendimientos, (definiéndolo como la rentabilidad económica de la organización y el aumento en ventas), la influencia del conocimiento relacionado va depender de qué tan inmerso esté el emprendedor en su organización, así como el tipo de emprendimiento que posea. Esto debido a que dependiendo del emprendimiento muchos de los emprendedores van sumando personal a su organización, con lo cual se van delegando funciones, en aspectos como las ventas y la rentabilidad económica no va depender estrictamente del conocimiento del emprendedor sino también del conocimiento de los colaboradores los cuales tienen intervención en este aspecto.

El trabajo, como cualquier otro de esta índole tiene limitaciones. Los resultados no son viables de inferir a toda la población de emprendedores dado el carácter cualitativo adoptado. No obstante, permite conocer habilidades que adquieren los emprendedores en su paso por las multinacionales y cómo estas afectan su desempeño, lo cual abre temas para futuras investigaciones, profundizar en las diferencias halladas por tipos de empresas, sectores, cultura organizacional de las mismas, entre otros. Asimismo, sería importante replicarlo en otros contextos diferentes al costarricense para apreciar diferencias producto de ello. Adicionalmente, se considera que proponer nuevas formas de medir el conocimiento relacionado, para poder ligarlo con otras variables organizacionales, podría ser otra ruta de investigación interesante.

\section{REFERENCIAS}

Brenes, L., Leiva, J. C., Chacón, C. L., Benavides, S. \& Govaere, V. (2009). Estado de la nacional de las Micro, Pequeñas y Medianas Empresas formales. San José, Costa Rica : EUNED.
Brush, C. G. \& Vanderwerf, P. A. (1992). A comparison of methods and sources for obtaining estimates of new venture. Journal of Business Venturing, 7, 157-170.

Calderón, M. (25 de abril de 2015). Relación entre conocimiento relacionado y desempeño en las Mipymes costarricenses (R. Villafuerte, entrevistador).

Cascante, P. (25 de abril de 2015). Relación entre conocimiento relacionado y desempeño en las Mipymes costarricenses (R. Villafuerte, entrevistador).

Castellani, D. \& Zanfei, A. (1998). Multinational growth and the creation of linkages with local firms: Evidence from the electronics industry. Cambridge Journal of Economics, 26, 1-26.

Clercq, D. D. \& Arenius, P. (2006). The Role of Knowledge in Business Start-up Activity. International Small Business Journal, 24, 339358.

Cortés, J. (14 de Abril de 2015). Relación entre conocimiento relacionado y desempeño en las Mipymes costarricenses (R. Villafuerte, entrevistador).

Coto, R. (31 de marzo de 2015). Relación entre conocimiento relacionado y desempeño en las Mipymes costarricenses (R. Villafuerte, entrevistador)

Davidsson, P. \& Honig, B. (2003). The role of social and human capital among. Journal of Business Venturing, 18, 301-331.

Dencker, J. C., Gruber, M. \& Shah, S. K. (2009). Pre-Entry Knowledge, Learning, and the Survival of New Firms. Organizationscience, 20, 516-537.

Gruber, M. (2007). Uncovering the value of planning in new venture creation: A process and contingency perspective. Journal of Business Venturing, 22(6), 782-807.

Hansen, M. T. (2002). Knowledge Networks: Explaining Effective. Organization Science, 3, 232-248. 
Hernández, R., Collado, C. \& Baptista, M. D. (2010). Metodología de la investigación (Quinta ed.). México D.F., México: McGrawHill.

Jaubert, W. (14 de abril de 2015). Relación entre conocimiento relacionado y desempeño en las Mipymes costarricenses (R. Villafuerte, entrevistador).

Leiva, J. C., Alegre, J. \& Monge, R. (2014). Los emprendedores surgidos de las empresas multinacionales de inversión extranjera directa: un estudio exploratorio en Costa Rica. Estudios Gerenciales, 30, 124-133.

Lopéz, V. A. \& Iglesias, S. (2006). percepciones directivas del recurso reputación. Estudio empírico de sus relaciones con el rendimiento empresarial. Cuadernos de Economía y Dirección de la Empresa, (28), 139-160.

Ministerio de Economía, I. (22 de Abril de 2013). PYMES Costa R/ca. Obtenido de http://www.pyme.go.cr/media/archivo/notici as/pcp-37-13.pdf

Monge, R. \& Torres, F. (20 de marzo de 2014). Comisión Asesora en Alta Tecnología. Obtenido de CAATEC: http://www.caatec.org/sitio1/images/stories/ publicaciones/entrepreneurship.pdf

Monge, R., Leiva, J. C. \& Alegre, J. (2011). Creación de empresas por parte de ex empleados de empresas multinacionales de alta tecnología en Costa Rica. Tec Empresarial, 5(3), 8-20.

Monge, R., Leiva, J. C. \& Alegre, J. (2012). Inversión extranjera directa, movilidad laboral y derrames de conocimiento en Costa Rica. Tecnología en Marcha, 25(5), 103-115.

Muñoz, A., Rosado, M. J. \& Vergara, N. (2012). Evaluación del impacto de las multinacionales Realidad de las empresas vinculadas vs. la percepción de las empresas no vinculadas. pensamiento y gestión, (32), 225-247.

Noel, T. W. \& West, P. (2009). The Impact of Knowledge Resources on New. Journal of Small Business Management, 47, 1-23.
Redondo, J. (10 de Abril de 2015). Relación entre conocimiento relacionado y desempeño en las Mipymes costarricenses (R. Villafuerte, entrevistador)

Rodríguez, A. (2009). Nuevas perspectivas para entender el emprendimiento empresarial. pensamiento \& gestión, 26, 94-119.

Rodríguez, A. (2009). Nuevas perspectivas para entender el emprendimiento empresarial. pensamiento \& gestión, 26, 94-119.

Sapienza, H. J., Parhankangas, A. \& Autio, E. (2009). Knowledge relatedness and post-spinoff growth. Journal of Business Venturing, 19, $809-829$

Sirias, C. (7 de abril de 2015). Relación entre conocimiento relacionado y desempeño en las Mipymes costarricenses (R. Villafuete, entrevistador).

Spencer, J. (2008). The impact of multinational enterprise strategy on indigenous enterprises: hotizontal spillovers and crowding. Academy of Management Review, 33(2), 341-361.

Tariverd, H. \& Venkatraman, N. (2005). Knowledge relatedness and the performence of multibusiness firms. Strategic Management Journal, 26, 97-119.

Weber, C. \& Weber, B. (2010). Social Capital and Knowledge Relatedness as Promoters of. International Studies of Management \& Organization, 40, 23-49.

Zahra, S. A., Ireland, D. \& Hitt, M. A. (2002). International Expansion by New Venture Firms: International Diversity, Mode of Market Entry, Technological Learning, and Performance. Academy of managment, 43(5), 925-950.

Zeledón, P. (8 de abril de 2015). Relación entre conocimiento relacionado y desempeño en las Mipymes costarricenses (R. Villafuerte, entrevistador) 